\title{
PULSED WIRE MAGNETIC FIELD MEASUREMENTS ON UNDULATOR U10P
}

\author{
T.C. Fan ${ }^{1,2}$, F.Y. Lin ${ }^{1}$, C.S. Hwang ${ }^{1}$, Ian C. Hsu ${ }^{2}$ \\ ${ }^{1}$ Synchrotron Radiation Research Center, Hsinchu, Taiwan \\ ${ }^{2}$ National Tsing Hua University, Hsinchu, Taiwan
}

\section{Abstract}

Several laboratories have used the pulsed wire method for magnetic field measurement. The acoustic wave distortion of the wire was observed. The limitations of this method were discussed. A pulsed wire system is under test in SRRC on an undulator U10p with a total length of $2 \mathrm{~m}$. In this paper the authors clarify the effect of wire imperfection and dispersion of acoustic wave, which are deemed to the main sources of the distortion and limitations. A strategy using thick wire is proposed to make this method reliable in precise measurement.

\section{INTRODUCTION}

The pulsed wire method was proposed [1] and applied to measuring wigglers and undulators in some groups [1-13]. One uses pulsed wire system to take the place of Hall probe system in the following situations:

a. One desires to make in situ measurements of wiggler magnetic fields so as to monitor the field error, calculate the radiation spectrum and cancel the wiggler steering errors.

b. One needs to speed the fine-tuning of wiggler field.

c. In the point measurement of mini-gap undulator the limited space cannot accommodate the support of the Hall probe.

d. Some dynamic behaviors are of concern. For example, pulsed magnets $[4,13]$ and current ramping of wiggler require a real time measurement system.

Because of the similar reasons, the Synchrotron Radiation Research Center (SRRC) plans to use the pulsed wire method to measure the small-gap superconducting wiggler. Before that, the pulse wire system is tested on a prototype undulator U10p with a total length of $2 \mathrm{~m}$ and a peak field of 1 Tesla. U10p has been well measured by Hall probe and long-loop systems. Fig. 1 shows the pulsed wire system. For the measurement of the $1^{\text {st }}$ integral of magnetic field, we use a digital function generator and a power transistor to generate a current pulse of $0.2 \mathrm{~ms}$ and 0.5 Ampere. The photo-coupler $\mathrm{H} 21 \mathrm{~A} 1$ is used to detect the horizontal displacement of the $\mathrm{Be}-\mathrm{Cu}$ wire due to the interaction between wire current and the magnetic field.

\footnotetext{
*fantc@srrc.gov.tw

${ }^{\dagger}$ However, to get a high $\mathrm{S} / \mathrm{N}$ ratio, the pulsed wire system also needs a large number of scans for average. Alternatively, digital filters might be applied if prudently enough.
}

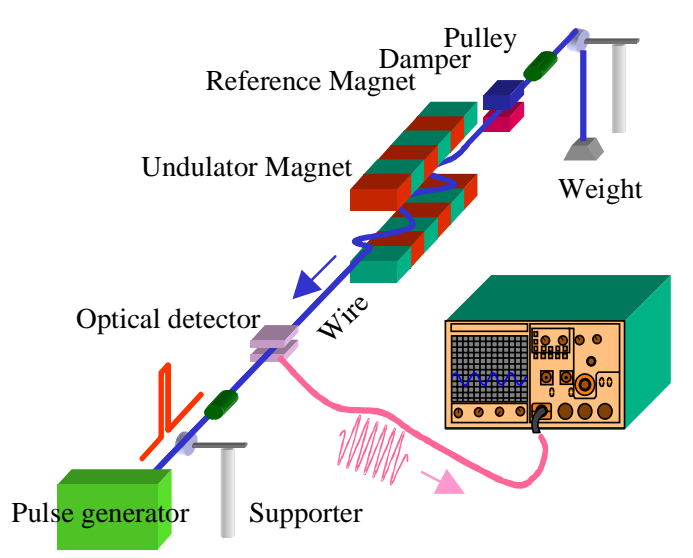

Figure 1:Simplified diagram of the pulsed-wire system.

Despite of the convenient setup, limitations of this method were discussed [1]. Moreover, some spurious components were confusing. They are: the distortions of the main signal (especially the beginning peak and ending peaks), the weak signals that arrive at the sensor after the main signal ("leading signal" for simplification) and the weak signals arrive before the main signal ("tail signal" for simplification) $[1,3,4,6]$. Fig. 2 illustrates these components. Some explanations were provided $[1,3,4]$.

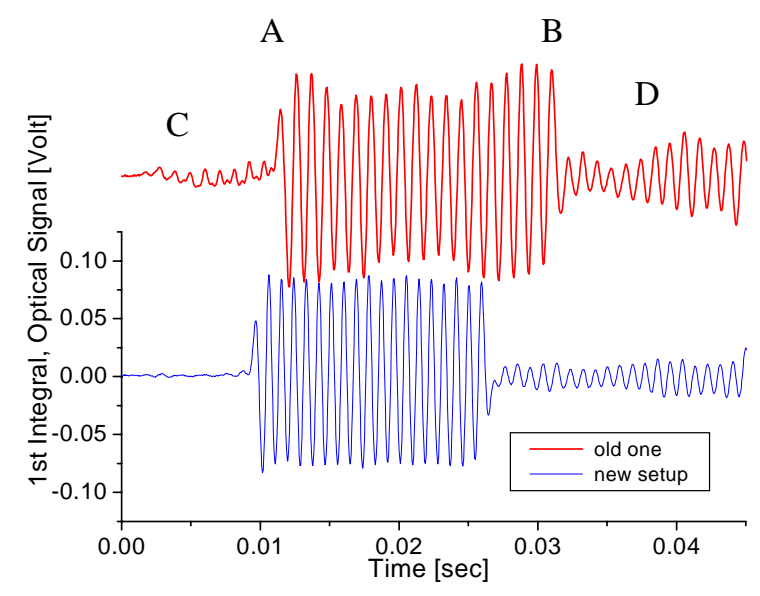

Figure 2: A typical result of pulsed wire measurement using thin wire. The upper curve has distortions in main undulator signal (A,B), leading signal (C) and tail signal (D). After readjustment and increasing the tension of the wire, one can elimiate some leading signal and get the lower curve. 
Through a series of systematic experiments, the authors believe that the tail signal comes from the imperfection of the wire and the other spurious signals mainly come from dispersion as the results of the finite stiffness of wires. To reach the accuracy of Hall probe system, these spurious signals should be eliminated. To put the tension of the wire to yield limit is a good way to reduce the dispersion effect (see also Fig. 2). Other approaches to reduce the wire imperfection and solve the dispersion will be proposed [15].

\section{WIRE IMPERFECTION}

The tail signals patterns differ from wire to wire even with the same diameter. They are reproducible for given wires. A typical waveform is shown in Fig. 2. The characteristic frequencies of these weak signals are the same as that of the main signal. This implies hints that they come from the interference of the reflection of main signal due to wire's imperfections.

Fig. 3 presents an experiment demonstrating how acoustic waves propagate at the source area. After pulse excitation, the original waveform was created and divided into two sub-waves propagating along opposite direction. Each sub-wave has one half of the amplitude of original waveform. We put an optical detector inside the undulator gap, i.e. at the source area. At the very beginning, two opposite waves overlap and detector gets a signal of full amplitude. After the two waves move away, the detector can see the sub-waves with half amplitude without overlap. One can also see that in the inset graph of Fig. 3, actually the wire displacement does not reach its full amplitude during the pulse period.

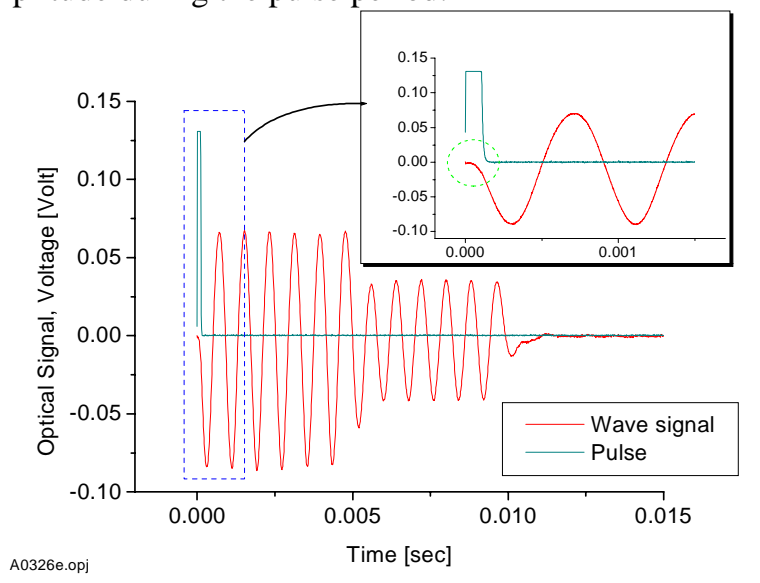

Figure 3: The time-dependent waveform detected by a photo detector inside the undulator gap.

When there are imperfections on the wire, one subwave will lose energy due to the reflection and get energy from the reflection of the other sub-wave. As a result, if the waveform of every period is of the same and only one imperfection is under consideration, the waveform would not alter when passing through the imperfections. However, if not, wave distortion and interference will occur and accumulate. Then we get a tail signal.
We purposely put some drops of wax or silicone oil purposely on a selected uniform wire to simulate the imperfection causing a significant tail signal. One example is shown in Fig.4

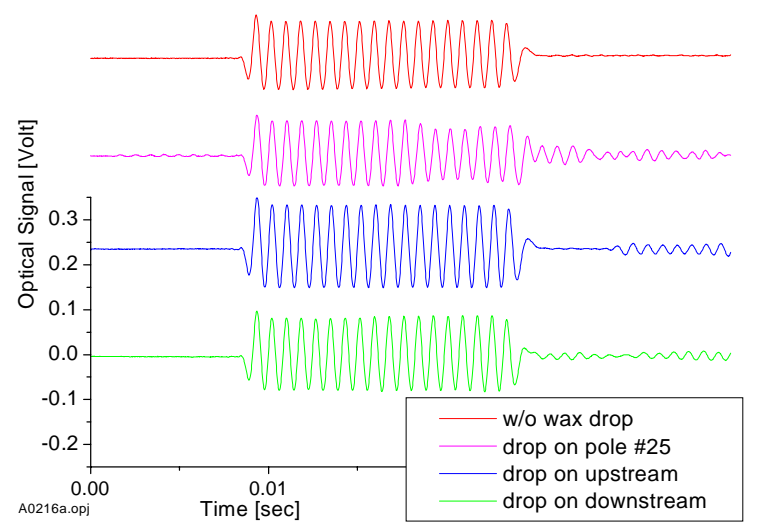

Figure 4: A simulation of imperfection effect of tail signals. From top to bottom, four curves represent the effect of manmade imperfections applied at the different position.

If this imperfection hypothesis is accepted, one can expect that a thick wire would not be sensitive to the impurity of the wire and have a better uniformity and less tail signal. We replace the wire with $125 \mu \mathrm{m}$ diameter by a $250 \mu \mathrm{m}$ one and indeed can reduce the signal. This agrees with the hypothesis of imperfection. An additional advantage of the thick wire is that the disturbance from airflow can be effectively reduced.

\section{DISPERSSION}

The transverse-motion equation of thin rods, well discussed in textbooks of mechanics [14], is

$$
-E I \frac{\partial^{4} x}{\partial z^{4}}+T \frac{\partial^{2} x}{\partial z^{2}}=\rho A \frac{\partial^{2} x}{\partial t^{2}},
$$

in which $E$ is modulus of elasticity, $I$ is moment of inertia, $T$ is the tension, $\rho$ is the mass density and $A$ is the area of cross section. Consider the propagation of harmonic waves in an infinite beam by considering the solution

$$
x=C e^{i(k x-\omega t)} .
$$

Substituting in differential equation gives

$k(\omega)= \pm \frac{T}{E I} \sqrt{-1 \pm \sqrt{1+\frac{\omega^{2} \rho A E I}{T^{2}}}}$.

For a circular cross section of diameter $d$,

$I=\frac{\pi d^{4}}{64}$.

Because of the dispersion function $k(\omega)$, each frequency component of a propagating wave will change the relative phase all the way and cause the interference in the time domain. Using a thick wire to reduce the imperfection effect, we can focus on the study of dispersion problem. First of all, we observe the distortion of the acoustic wave 
at different distance from the wave source excited by a single-pole magnet. Fig. 5 shows that the distortion of the main signal and leading signal are getting obvious when detector distance increases. The mathematical simulation based on the dispersion function $k(\omega)$ is in good agreement with this observation [15].

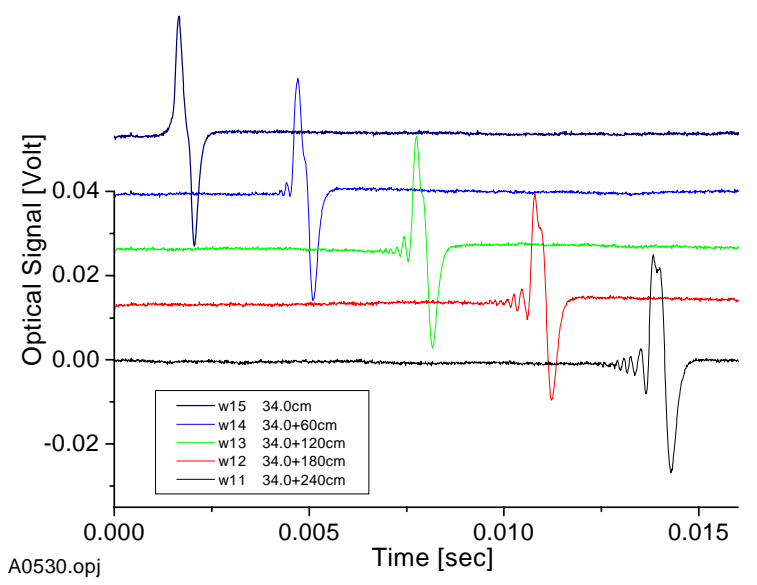

Figure 5: The observation of wave distortion at different distance from source.

The increase of wire tension will speed the wave propagation and reduce the phase difference. This definitely reduces the dispersion-involved distortion. Fig. 6 presents a comparison between Hall probe and the pulsed wire measurement with high tension and thick wire. There is small but still appreciable difference at the first two and the last two poles. This difference is pulsewidth and tension dependent.

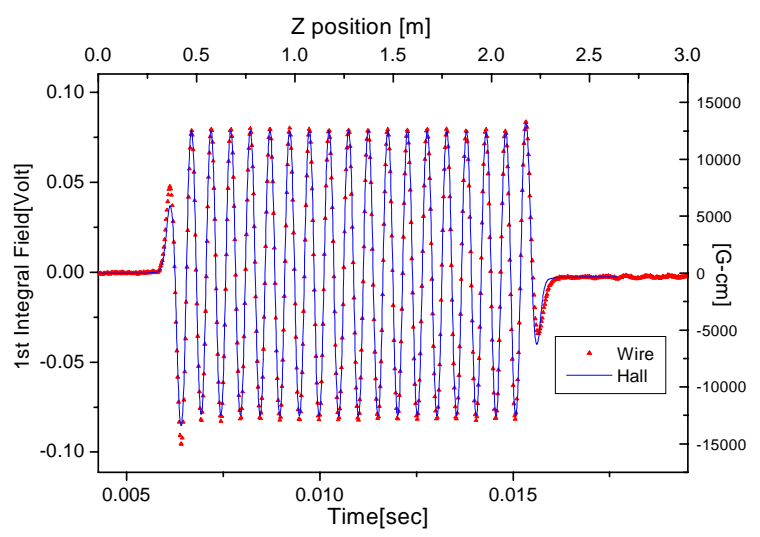

Figure 6: Results of magnetic field measurement by Hall probe and pulsed wire method under higher tension.

\section{SUMMARY}

The authors clarify that the wire imperfection will cause the tail signal and dispersion function will cause leading signal. Using thick wire we can take away the tail signal distortion. Next, the authors will cancel the effect of the dispersion to recover the wave from distortion [15].

\section{REFERENCES}

[1] R. W. Warren, "Limitations on the use of the pulsedwire field measuring technique", Nucl. Instr. and Meth., A272 (1988) 257

[2] R. B. Feldman and R. W. Warren, "Fully automatic wiggler-field test and correction", Nucl. Instr. and Meth., A296 (1990) 619-623

[3] Daryl W. Preston Roger W. Warren, "Wiggler field measurements and corrections using the pulsed wire technique", Nucl. Instr. and Meth., A318 (1992) 794797

[4] Roger W. Warren and Daryl W. Preston, "Field measurement in pulsed microwigglers", $\left(13^{\text {th }}\right.$ Int. Free Electron laser Conf., Santa Fe, USA, 1991) Nucl. Instr. and Meth. A318 (1992) 818

[5] O. Shahal and R. Rohatgi, "Pulsed Wire Magnetic Field Measurements on a $4.3 \mathrm{~m}$ long wiggler", Nucl. Instr. and Meth., A285 (1989) 299-302

[6] O. Shahal, B.V. elkonin and J.S. Sokolowski, "Dispersion interference in the pulsed-wire measurement method", Nucl. Instr. and Meth., A296 (1990) 588-591

[7] S. M. Wallace, W.B. Colson, G.R. Neil, L. Harwood, "Magnetic field error measurement of the CEBAF (NIST) wiggler using the pulsed wire method", Nucl. Instr. and Meth. (14 ${ }^{\text {th }}$ international FEL confer.), A331 (1993) 759-762

[8] A. A. Varfolomeev et., "Undulator magnetic field measurements with the wire deflection", Nucl. Instr. and Meth., A341 (1994) 470-472

[9] A. A. Varfolomeev et. "Improved wire deflection method for magnetic field measurements in long undulators", Nucl. Instr. and Meth., A358 (1995) ABS46-47

[10] A. A. Varfolomeev et. "Wire method for magnetic field measurements in long undulators", Nucl. Instr. and Meth., A359 (1995) 93-96

[11] P.V. Bousine, S.V. Tolmachev, A. A. Varfolomeev, "Detailed analysis of pulsed-wire technique accuracy", Nucl. Instr. and Meth., A393 (1997) 414-418

[12] N.S. Osmanov, S.V. Tolmachev, A. A. Varfolomeev, "Further development of the pulsed wire technique for magnetic field and focusing strength measurements in long undulators", Nucl. Instr. and Meth., A407 (1998) 443-447

[13] Mateau Fabrice et. "Improvements of the PulsedWire Method to Measure Undulators", MT -16 (1999), IEEE Transaction on Applied Superconductivity, vol. 10, no.1, March 2000, 1443-1446

[14] Karl F. Graff, "Wave Motion in Elastic Solids", Ohio State University Press, 1975

[15] T.C. Fan, to be submitted to SRI2001 